\title{
Production cross sections for intermediate mass fragments from dynamical and statistical decay of projectile-like fragments in ${ }^{124} \mathrm{Sn}+{ }^{64} \mathrm{Ni}$ and ${ }^{112} \mathrm{Sn}+{ }^{58} \mathrm{Ni}$ collisions at $35 \mathrm{~A} \mathrm{MeV}$
}

\author{
P. Russotto, ${ }^{1,}{ }^{*}$ E. De Filippo, ${ }^{1}$ A. Pagano, ${ }^{1}$ L. Acosta,${ }^{2}$ L. Auditore,${ }^{3}$ V. Baran,${ }^{4}$ I. Berceanu,${ }^{5}$ B. Borderie, ${ }^{6}$ R. Bougault ${ }^{7}$ \\ M. Bruno, ${ }^{8}$ T. Cap, ${ }^{9}$ G. Cardella, ${ }^{1}$ S. Cavallaro,,${ }^{2,10}$ M. B. Chatterjee, ${ }^{11}$ A. Chbihi, ${ }^{12}$ M. Colonna, ${ }^{2}$ M. D'Agostino, ${ }^{8}$ \\ M. Di Toro, ${ }^{2,10}$ L. Francalanza, ${ }^{2,10}$ J. Frankland, ${ }^{12}$ E. Galichet, ${ }^{6,13}$ W. Gawlikowicz, ${ }^{14}$ E. Geraci, ${ }^{1,10}$ B. Gnoffo, ${ }^{1,10}$
} A. Grzeszczuk, ${ }^{15}$ P. Guazzoni ${ }^{16}$ S. Kowalski, ${ }^{15}$ G. Lanzalone,${ }^{2,17}$ G. Lanzanò,${ }^{1,}$ N. Le Neindre,${ }^{7}$ I. Lombardo, ${ }^{18}$ C. Maiolino, ${ }^{2}$ T. Minniti, ${ }^{1}$ E. V. Pagano,${ }^{2,10}$ M. Papa,${ }^{1}$ E. Piasecki, ${ }^{19,20}$ S. Pirrone, ${ }^{1}$ R. Płaneta, ${ }^{21}$ G. Politi, ${ }^{1,10}$ F. Porto,${ }^{2,10}$ L. Quattrocchi, ${ }^{3}$ M. F. Rivet, ${ }^{6}$ E. Rosato, ${ }^{18}$ F. Rizzo, ${ }^{2,10}$ K. Schmidt,${ }^{15}$ K. Siwek-Wilczyńska, ${ }^{9}$ I. Skwira-Chalot, ${ }^{9}$ A. Trifirò, ${ }^{3}$ M. Trimarchi, ${ }^{3}$ G. Verde, ${ }^{1}$ M. Vigilante, ${ }^{18}$ J. P. Wieleczko, ${ }^{12}$ J. Wilczyński, ${ }^{20}$ L. Zetta, ${ }^{16}$ and W. Zipper ${ }^{15}$

${ }^{1}$ INFN, Sezione di Catania, Italy

${ }^{2}$ INFN, Laboratori Nazionali del Sud, Catania, Italy

${ }^{3}$ INFN, Gruppo Collegato di Messina and Dip. di Fisica, Univ. di Messina, Italy

${ }^{4}$ Physics Faculty, University of Bucharest, Romania

${ }^{5}$ National Institute of Physics and Nuclear Engineering "Horia Hulubei," Bucharest, Romania

${ }^{6}$ Institut de Physique Nucléaire, CNRS/IN2P3, Université Paris-Sud 11, Orsay, France

${ }^{7}$ LPC Caen, ENSICAEN, University of Caen, CNRS/IN2P3, Caen France

${ }^{8}$ INFN, Sezione di Bologna and Dipartimento di Fisica, Univ. di Bologna, Italy

${ }^{9}$ Faculty of Physics, University of Warsaw, Warsaw, Poland

${ }^{10}$ Dipartimento di Fisica e Astronomia, Univ. di Catania, Catania, Italy

${ }^{11}$ Saha Institute of Nuclear Physics, Kolkata, India

${ }^{12}$ GANIL (DSM-CEA/CNRS/IN2P3), Caen, France

${ }^{13}$ Conservatoire National des Arts et Métiers, F-75141 Paris Cedex 03, France

${ }^{14}$ Cardinal Stefan Wyszyński University, Warsaw, Poland

${ }^{15}$ Institute of Physics, University of Silesia, Katowice, Poland

${ }^{16}$ INFN, Sezione di Milano and Dipartimento di Fisica, Univ. di Milano, Italy

17 "Kore" Università di Enna, Enna, Italy

${ }^{18}$ INFN, Sezione di Napoli and Dipartimento di Fisica, Univ. di Napoli, Italy

${ }^{19}$ Heavy Ion Laboratory, University of Warsaw, Warsaw, Poland

${ }^{20}$ National Centre for Nuclear Research, Swierk/Warsaw, Poland

${ }^{21}$ M. Smoluchowski Institute of Physics, Jagellonian Univ., Cracow, Poland

(Received 24 November 2014; published 21 January 2015)

\begin{abstract}
Background: In heavy-ion collisions at intermediate energies the production of light charged particles and intermediate mass fragments (IMFs) is due to different reaction mechanisms and different time scales, ranging from fast dynamical processes to statistical emission from the equilibrated system.

Purpose: We compare the IMF statistical and dynamical emission probabilities in collisions of a neutron-rich ${ }^{124} \mathrm{Sn}+{ }^{64} \mathrm{Ni}$ system and a neutron-poor ${ }^{112} \mathrm{Sn}+{ }^{58} \mathrm{Ni}$ system at the laboratory energy of $35 \mathrm{~A} \mathrm{MeV}$.

Method: The IMFs production mechanism in semiperipheral reactions has been investigated in our previous works. In this paper, the analysis is expanded for the same set of data and production cross sections have been evaluated for dynamical and statistical emission in a coherent way for light and heavy fragments.

Results: The data analysis has evidenced a strong competition between dynamical and statistical emission mechanisms. Probability of the dynamical emission of IMFs is strongly influenced by the $(N / Z)$ ratio of the colliding system.

Conclusions: It is demonstrated that the statistical emission is equally probable for the two systems, while the dynamical emission is enhanced for the neutron-rich system, especially for heavy fragments $(Z \geqslant 6)$. The observed effect points at a high sensitivity of the prompt-dynamical emission to the $(N / Z)$ ratio of a given system.
\end{abstract}

DOI: 10.1103/PhysRevC.91.014610

PACS number(s): 25.70.Mn, 25.70.Pq

\section{INTRODUCTION}

A characteristic experimental signature of heavy-ion reactions in the Fermi energy domain $(20 \mathrm{~A}-100 \mathrm{~A} \mathrm{MeV})$ is the

\footnotetext{
*russotto@lns.infn.it
}

${ }^{\dagger}$ Deceased. abundant emission of relatively light fragments, the so-called intermediate mass fragments (IMFs), with atomic number $Z$ in the range of values $3 \leqslant Z \leqslant 20$. Typically, IMFs show a broad velocity distribution ranging from velocities of target-like fragments (TLF) to much higher velocities of projectile-like fragments (PLF), indicating different reaction mechanisms and time scales, from dynamical and pre-equilibrium emissions to 
statistical decay of excited systems at equilibrium. Invoked mechanisms are projectile or target fission, breakup and fragmentation, neck emission, and multifragmentation. $\mathrm{Nu}$ clear multifragmentation is the typical mechanism in central collisions. In this process the high multiplicity of emitted IMFs was linked to a first-order liquid-gas phase transition in nuclear matter [1-3]. In semiperipheral reactions the production of IMFs and light charged particles in the velocity region intermediate between the PLF and TLF velocities (midrapidity source) has been experimentally observed and the characteristic features of a dynamical process (not related to a statistical emission from equilibrated sources) have been clearly evidenced in several works (see Refs. [4-7] and references therein). In the "neck" fragmentation scenario light IMFs at midrapidity are emitted through a stochastic rupture or fragmentation of a piece of dilute nuclear matter ("neck") linking the quasiprojectile and the quasitarget in the early phase of the binary collision $(\sim 100 \mathrm{fm} / \mathrm{c})$ [4]. A recent theoretical analysis in the frame of a dynamical transport model [8] suggests a continuous transition from the ternary neck emission mechanism at semiperipheral collisions to the high multiplicity multifragmentation processes at near-central collisions.

Many experimental results have shown an enhancement [9-15] of production of neutron-rich fragments at midrapidity as compared with expectations of the statistical decay.

In a recent paper [12], a strong sensitivity of the isotopic content of the fragments to the alignment properties and timescale of their emission pattern has been demonstrated and linked to the symmetry term of the nuclear equation of state (EOS), enhancing the relevant role of dynamical constraints in such studies [16].

The mechanism of emission of midrapidity IMFs in semiperipheral $\mathrm{Sn}+\mathrm{Ni}$ dissipative collisions at laboratory energy of $35 \mathrm{~A} \mathrm{MeV}$ has been carefully investigated using the forward part of the $4 \pi$ CHIMERA multidetector [17,18]. A clear signature of an emission chronology correlated to the IMF size was demonstrated [12,19-23]. In particular, it was found that light IMFs $(Z \lesssim 9)$ are likely to be emitted fast, within $120 \mathrm{fm} / \mathrm{c}$, during the mutual neck-reseparation stage of primary PLF* and TLF* [19,20,22], while the emission of heavy IMFs $(Z \gtrsim 9)$ was found to happen at the latest stage of the neck expansion process and associated with an asymmetric mass splitting of PLF* (“dynamical fission") [21-25]. Thus a two step (sequential) reaction was evidenced: The dissipative scattering with excitation of primary nuclei (PLF*-TLF*) followed by fast nonequilibrated fissionlike splitting. A similar process of aligned nearly symmetric breakup of PLF* and/or TLF* in collisions of a heavy nuclear system $\mathrm{Au}+\mathrm{Au}$ was also observed at lower bombarding energies of $15 A \mathrm{MeV}$ [26-28].

The main experimental signature of the dynamical asymmetric fission of PLF* in two massive fragments are (a) the lightest of the two massive fragments is emitted preferentially between its heavy PLF partner and the TLF, thus resulting in an aligned three-body configuration, and (b) the relative velocity of the two correlated fragments (PLF and IMF) is somewhat larger (by about 20\%) than the value resulting from the Viola systematics predicted for equilibrated fissionlike decay (see below, Sec. II). The analysis suggested that the dynamical fission occurs later than $\sim 120 \mathrm{fm} / \mathrm{c}$ after the the neck reseparation of PLF* and TLF*, but before the PLF* has enough time to perform a full rotation and loose memory of the PLF*-TLF* $^{*}$ reseparation axis. This experimental picture of IMF emission chronology is supported both by stochastic mean field (SMF) [29,30] and molecular dynamics (CoMD-II) [22] calculations. In addition to these fast-dynamical mechanisms, much slower de-excitations [31] of equilibrated PLF* and TLF*, characterized by isotropic angular distributions, contribute to the IMF emission.

In previous studies it was already seen that for the systems and energy investigated in this paper a steady increase of the violence of the collision leads to multiple IMF emission: The reaction mechanism becomes more complex (coexistence of different mechanisms) and evolves toward the multifragmentation of a unique source of emission [32,33].

In this paper the previous analysis of Ref. [23] is extended by enlarging by about a factor of 2 the impact parameter window of the collision toward more dissipative collisions, giving a unified, charge by charge, evaluation of cross sections of the observed IMFs from atomic number $Z=3$ up to $Z=22$. Thus, we have compared IMF emission probability in the neutron-rich ${ }^{124} \mathrm{Sn}+{ }^{64} \mathrm{Ni}$ and neutron-poor ${ }^{112} \mathrm{Sn}+{ }^{58} \mathrm{Ni}$ systems. We have carefully evaluated the production cross section for statistical and dynamical emission of fragments for both light and heavy IMFs.

\section{EXPERIMENTAL RESULTS}

The experiment was performed at the INFN-LNS Superconducting Cyclotron of Catania (Italy), bombarding thin $\left(\approx 300 \mu \mathrm{g} / \mathrm{cm}^{2}\right)$ self-supporting ${ }^{64} \mathrm{Ni}$ and ${ }^{58} \mathrm{Ni}$ targets with $35 \mathrm{~A} \mathrm{MeV}{ }^{124} \mathrm{Sn}$ and ${ }^{112} \mathrm{Sn}$ beams, respectively. Reaction products were detected with the forward part of the $4 \pi$ multidetector CHIMERA [19] that is constituted by $688 \mathrm{Si}(\approx 300$ $\mu \mathrm{m})$-CsI(Tl) telescopes arranged in 18 rings and covering the angular range between $1^{\circ}$ and $30^{\circ}$. Particles punching through the silicon detectors were identified in charge (and for light ions up to $Z \leqslant 9$ also in mass number) by using the $\Delta E-E$ technique. Light charged particles (LCP) of atomic numbers $Z \leqslant 2$ were identified isotopically in the pulse shape analysis (fast-slow technique) of CsI(Tl) signals. Moreover, information on the mass of particles stopped in silicon detectors was obtained via the time-of-flight (TOF) technique using the timing signal from silicon detectors with respect to the timing of the high-frequency signal from the cyclotron. The TOF technique provides direct velocity measurement for heavy ions of $Z>2$. More details about the experimental methods can be found in our previous works on these reactions and references therein [12,20,21,23]. Reaction simulation codes (see Ref. [34] as an example) and experimental correlations show [35] that both size and velocity of PLF* decrease with increasing collision centrality. In this paper we deal with semiperipheral collisions. Therefore we selected events with PLF residues having atomic numbers $Z \gtrsim 20$ and parallel to the beam velocity components (in laboratory reference frame) $V_{\text {par }} \gtrsim 6 \mathrm{~cm} / \mathrm{ns}$. The selection was performed by applying a contour gate in the $Z$ vs $V_{\text {par }}$ two-dimensional plot as shown in Figs. 1(a) and 1(c) for the neutron-rich ${ }^{124} \mathrm{Sn}+{ }^{64} \mathrm{Ni}$ system 

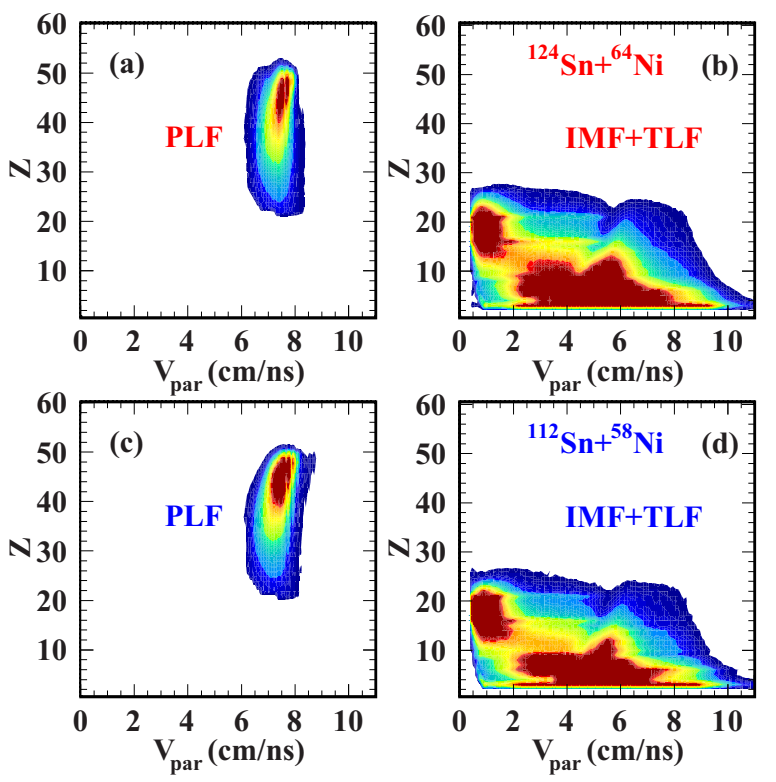

FIG. 1. (Color online) Panels (a) and (c): Atomic number $Z$ vs parallel-to-the-beam velocity $V_{\text {par }}$ plots for PLF residues in the ${ }^{124} \mathrm{Sn}$ $+{ }^{64} \mathrm{Ni}$ and ${ }^{112} \mathrm{Sn}+{ }^{58} \mathrm{Ni}$ systems, respectively. Panels (b) and (d): $Z$ vs $V_{\text {par }}$ plots for IMFs and TLFs in coincidence with PLF residues for the same systems.

and neutron-poor ${ }^{112} \mathrm{Sn}+{ }^{58} \mathrm{Ni}$ system, respectively. This is a generalization of the selection of ternary events described in Sec. III.A of Ref. [20] (see Fig. 2 of that reference for a comparison).

The $Z-V_{\text {par }}$ two-dimensional plots of IMFs detected in coincidence with the PLF, as well as TLF residues, are shown in Figs. 1(b) and 1(d).

In most of the selected events $Z$ and $V_{\text {par }}$ of the PLF are $Z \sim 45$ and $V_{\text {par }} \sim 7.5 \mathrm{~cm} / \mathrm{ns}$ (slightly below the beam velocity of $\approx 8 \mathrm{~cm} / \mathrm{ns}$ ). By estimating the impact parameter from the total charged-particle multiplicity using the method of Cavata et al. [36], the selected data set for both $\mathrm{Sn}+\mathrm{Ni}$ systems can be associated to a range of the reduced impact parameter $b / b_{\max }=b_{\text {red }}>0.4$, where $b_{\max }$ corresponds to the total (geometrical) nucleus-nucleus cross section.

The multiplicity of IMFs detected in coincidence with the PLF in the two $\mathrm{Sn}+\mathrm{Ni}$ systems, normalized to the number of selected events, is compared in Fig. 2(a). The TLF residues, if any, have been excluded by applying a contour gate in the TLF region ( $Z \sim 20$ and $V_{\text {par }} \sim 1 \mathrm{~cm} / \mathrm{ns}$ ) of Figs. 1(b) and 1(d). Data of Fig. 2 have been obtained by taking into account the detection efficiency of the experimental apparatus, using the HIPSE code [37] as an event generator and a software replica of CHIMERA multidetector [38].

Events with IMF multiplicity equal to zero correspond to "binary events" in which only LCP and neutrons (no detected with our apparatus) accompanying the PLF-TLF binary partners have been produced. These events (IMF multiplicity = 0 ) are more probable for the neutron-poor system. However, this effect, associated with the most peripheral collisions, can be influenced by the difference of the grazing angle between the two systems (see below) and the "blindness" to neutrons and energetic protons of our triggering system, based on silicon detector multiplicity. Note that the IMF emission (IMF multiplicity $\geqslant 1)$ is enhanced in the neutron-rich ${ }^{124} \mathrm{Sn}+{ }^{64} \mathrm{Ni}$ system.

In Fig. 2(b) we display values of the cross section associated with the IMF multiplicity. The same effect, i.e., the enhancement of the IMF emission for the neutron-rich system is found. The absolute cross sections have been obtained by normalization to the singles' counts of elastic scattering measured with a telescope covering the polar angle range $\vartheta_{\text {lab }}=1^{\circ}-1.8^{\circ}$ (see Fig. 4 of Ref. [39] for details). According to Ref. [40], values of the grazing angle are $\vartheta_{\text {grazing }}=2.18^{\circ}$ and $2.5^{\circ}$ for the neutron-rich and neutron-poor systems, respectively. An error of about $10 \%$ for this normalization procedure is expected. Finally Fig. 2(c) presents the ratio of the two probabilities shown in Fig. 2(a) versus the IMF multiplicity. Figure 2(c) clearly shows that, for the class of semiperipheral events discussed here, the ratios of probability for the ${ }^{124} \mathrm{Sn}+{ }^{64} \mathrm{Ni}$ and ${ }^{112} \mathrm{Sn}+{ }^{58} \mathrm{Ni}$ reactions steadily increases with the IMF multiplicity. Obviously, the neutron enrichment of the colliding systems enhances the probability of multiple cluster formation.
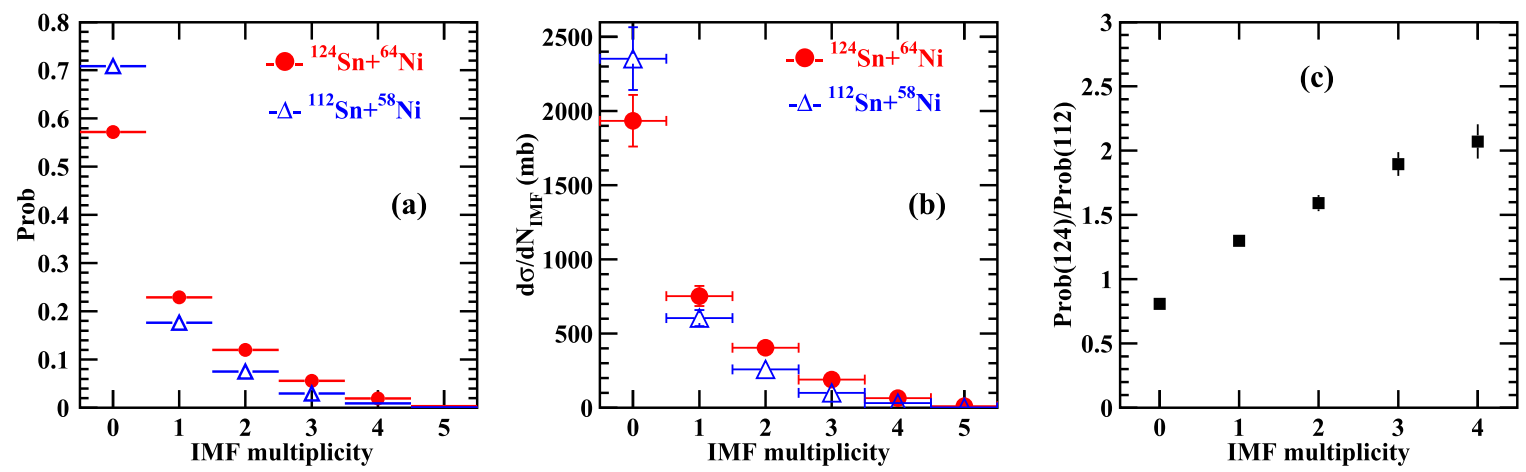

FIG. 2. (Color online) (a) Probabilities of different IMF multiplicities (in coincidence with PLF residues) normalized to the number of selected events for the ${ }^{124} \mathrm{Sn}+{ }^{64} \mathrm{Ni}$ (full circles) and ${ }^{112} \mathrm{Sn}+{ }^{58} \mathrm{Ni}$ (empty triangles) systems. (b) Cross sections corresponding to different IMF multiplicities for the same systems. (c) Ratios of probabilities displayed in panel (a) for the ${ }^{124} \mathrm{Sn}+{ }^{64} \mathrm{Ni}$ and ${ }^{112} \mathrm{Sn}+{ }^{58} \mathrm{Ni}$ reactions, plotted as a function of the IMF multiplicity. All the plots are corrected for detection efficiency. 

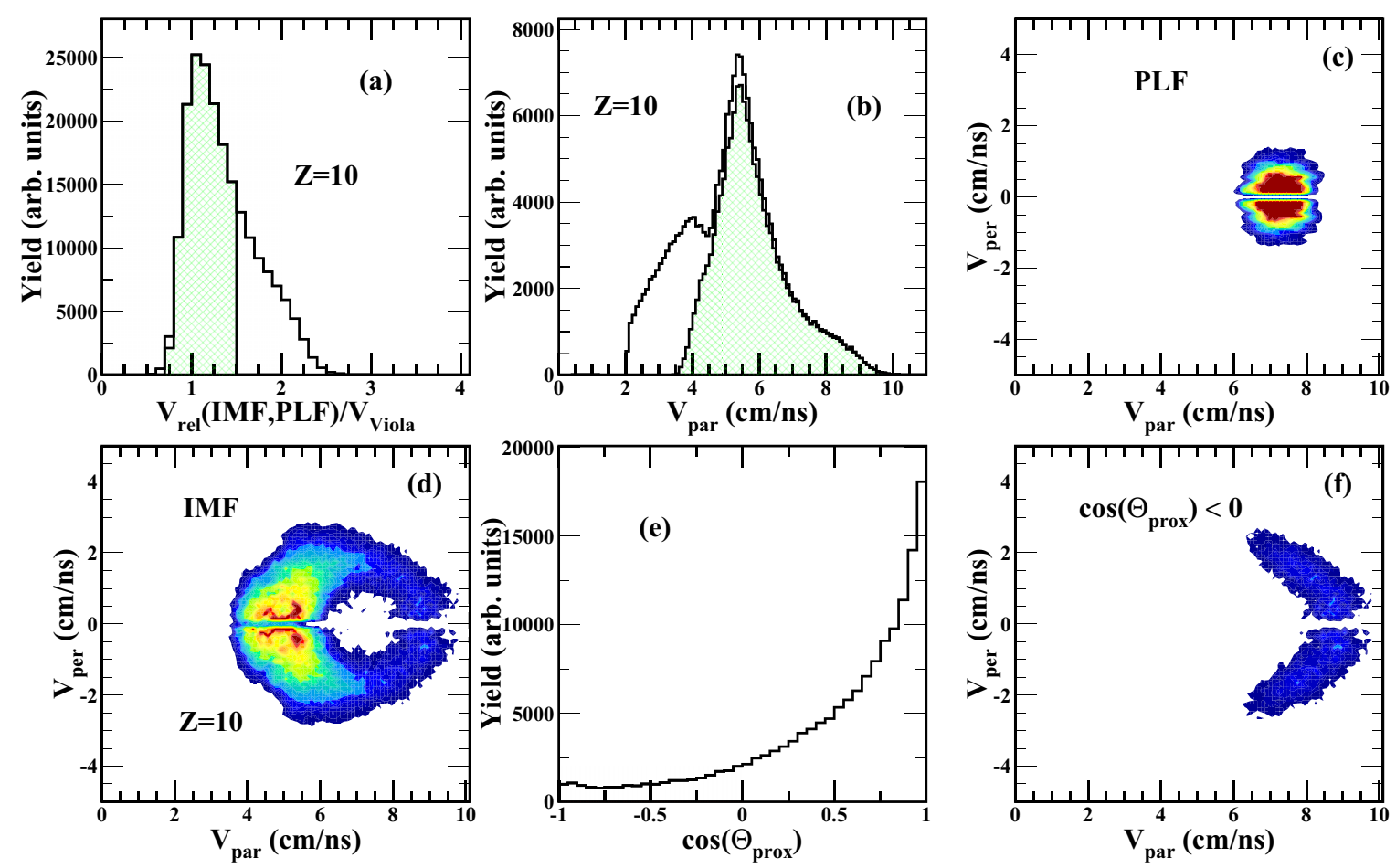

FIG. 3. (Color online) Plots for the ${ }^{124} \mathrm{Sn}+{ }^{64} \mathrm{Ni}$ system: (a) Relative velocity between PLF and IMF for IMFs of $Z=10$, normalized to the velocity given by the Viola systematics (black line). Dashed area represents events satisfying condition of Eq. (1). (b) Distribution of the velocity component $V_{\text {par }}$ (parallel to the beam axis) in the laboratory reference frame for IMFs of $Z=10$ (black line). Dashed area represents events satisfying condition of Eq. (1). (c) Invariant cross-section plot ( $V_{\text {par }}$ vs $V_{\text {per }}$, where $V_{\text {per }}$ is the perpendicular component) for PLF residues. (d) Invariant cross-section plot for IMFs of $Z=10$. (e) Angular distribution of IMFs of $Z=10$ originated from PLF* splitting (full black line). (f) Invariant cross-section plot for IMFs of $Z=10$ and $\cos \left(\theta_{\text {prox }}\right)<0$.

In order to investigate the cause of the difference in the IMF emission probabilities between the two systems, we have estimated the contribution of both the statistical-equilibrated and dynamical-emission mechanisms. We limited this analysis to a class of events with the IMF multiplicity equal to one, i.e., to events of ternary splitting of the colliding system. The procedure was as follows: First, the IMFs emitted from TLF have been removed by rejecting events with the relative velocity between PLF and IMF, $V_{\text {rel }}$ (PLF,IMF), larger than 1.5 times the relative velocity due to mutual PLF-IMF Coulomb repulsion, $V_{\text {Viola }}$, evaluated using the Viola systematics [41]. The choice of this cut [see, e.g., Fig. 3(a)] has been guided by results of our previous analysis of relative velocity correlations between IMF and PLF or IMF and TLF (see Fig. 7 of Ref. [20] and simulations reported in the Appendix of Ref. [21]). As an example illustrating the analysis of the ${ }^{124} \mathrm{Sn}+{ }^{64} \mathrm{Ni}$ reaction, Figs. 3(a) and 3(b) show the $V_{\text {rel }}(\mathrm{PLF}, \mathrm{IMF}) / V_{\text {Viola }}$ and $V_{\mathrm{par}}$ distributions (black lines) for the IMF charge $Z=10$. Events satisfying the condition

$$
V_{\text {rel }}(\mathrm{PLF}, \mathrm{IMF}) / V_{\text {Viola }} \leqslant 1.5
$$

are represented in Figs. 3(a) and 3(b) by the dashed area. Data displayed in Figs. 3(c)-3(f) also satisfy the condition of Eq. (1). It is seen from Fig. 3(b) that for IMF $=10$ the $V_{\text {par }}$ distribution is roughly composed of two components, one centered around $V_{\mathrm{par}} \approx 3.5 \mathrm{~cm} / \mathrm{ns}$, probably related to the TLF* fragmentation [20,22], and another one centered around $V_{\mathrm{par}} \approx 5.5 \mathrm{~cm} / \mathrm{ns}$, probably related to the $\mathrm{PLF}^{*}$ decay.
The applied condition [Eq. (1)] selects the latter component. Figures 3(c) and 3(d) show the $V_{\text {par }}$ vs $V_{\text {per }}$ Galilean-invariant cross-section plots for PLFs and IMFs of $Z=10$, respectively. In the $V_{\text {par }}$ vs $V_{\text {per }}$ distribution of IMFs we see a structure reminiscent of the Coulomb ring, centered around the centroid of the PLF parallel velocity. This structure can be related to the PLF* fissionlike decay mechanism. At somewhat lower parallel velocities around $V_{\mathrm{par}} \sim 4 \mathrm{~cm} / \mathrm{ns}$, one can see some midvelocity fragments outside the ringlike structure, originated from a fast dynamical process reminiscent of the neck-rupture mechanism. The pattern of the $V_{\text {par }}$ vs $V_{\text {per }}$ correlation observed for emission of heavier fragments $(Z \gtrsim$ 9) allows us to presume the concept of dynamical fission, although this process could also be interpreted as a delayed emission from the neck zone connecting the interacting target and projectile.

In the statistical model, a forward-backward symmetric distribution of IMFs within the Coulomb ring is expected. In Fig. 3 one can note that IMFs populate preferentially the low-velocity side of the Coulomb ring. This means that they are backward emitted in the reference frame of the primary PLF*. This is the main signature of the dynamical emission. The entire distribution can be interpreted as a superposition of the forward-backward symmetric distribution and the remaining asymmetric component. In order to estimate weights of the two components, we have calculated angular distributions of IMFs plotted as a function of $\cos \left(\theta_{\text {prox }}\right)$, where $\theta_{\text {prox }}$ is the angle (measured in the rest frame of the reconstructed PLF*) 

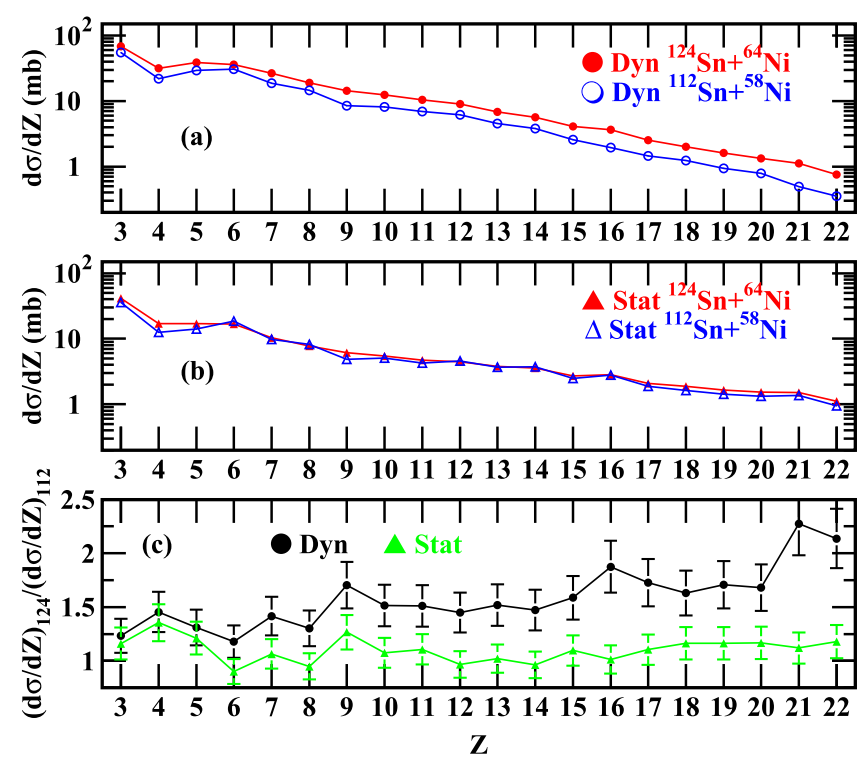

FIG. 4. (Color online) (a) Dynamical IMF emission cross sections (in mbarn) for the ${ }^{124} \mathrm{Sn}+{ }^{64} \mathrm{Ni}$ (full circles) and ${ }^{112} \mathrm{Sn}+{ }^{58} \mathrm{Ni}$ (empty circles) systems. (b) Statistical IMF emission cross sections (in mbarn) for the ${ }^{124} \mathrm{Sn}+{ }^{64} \mathrm{Ni}$ (full triangles) and ${ }^{112} \mathrm{Sn}+{ }^{58} \mathrm{Ni}$ (empty triangles) systems. (c) Ratios of the cross sections for the two $\mathrm{Sn}+\mathrm{Ni}$ systems, neutron rich over neutron poor, for the dynamical (circles) and statistical emission (triangles). The cross sections in panels (a) and (b) and the ratios in panel (c) are plotted as a function of $Z$ of the IMF.

between the PLF* velocity vector (along the TLF*-PLF* separation axis) and the velocity vector of the heaviest fragment between the two originating from the PLF* decay. In case of the statistical PLF* splitting a symmetric distribution with respect to $\cos \left(\theta_{\text {prox }}\right)=0$ is expected. The angular distribution for IMFs of $Z=10$ is shown in Fig. 3(e). A strong peak at $\cos \left(\theta_{\text {prox }}\right)=$ +1 dominates this distribution. The observed peak corresponds to the breakup along the TLF*-PLF* separation axis. The IMF fragment is then backward emitted. Applying the method of Ref. [25], the statistical contribution was determined as the maximum possible component of the angular distribution that is symmetric around $\cos \left(\theta_{\text {prox }}\right)=0$. The remaining part was then assigned as the "dynamical" contribution. As an example, Fig. 3(f) shows the $V_{\text {par }}$ vs $V_{\text {per }}$ distribution for IMFs of $Z=10$, gated by the condition $\cos \left(\theta_{\text {prox }}\right)<0$.

The dynamical and statistical components of the IMF emission cross sections, separated as described above, are plotted as a function of $Z$ of the IMF in Figs. 4(a) and 4(b), respectively. The cross sections are given for both nuclear systems, ${ }^{124} \mathrm{Sn}+{ }^{64} \mathrm{Ni}$ and ${ }^{112} \mathrm{Sn}+{ }^{58} \mathrm{Ni}$. While the statistical component is approximately the same for both nuclear systems, the dynamical component is definitely larger for the neutron-rich system. This fact is quantitatively illustrated in Fig. 4(c), where ratios of the dynamical and statistical components for both systems, $\sigma_{\text {neutron rich }} / \sigma_{\text {neutron poor }}$, are displayed. It is seen that the ratio of the statistical contributions stays approximately constant at a value of about 1.1, while the ratio of the dynamical components steadily increases with increasing $Z$ of the IMF,
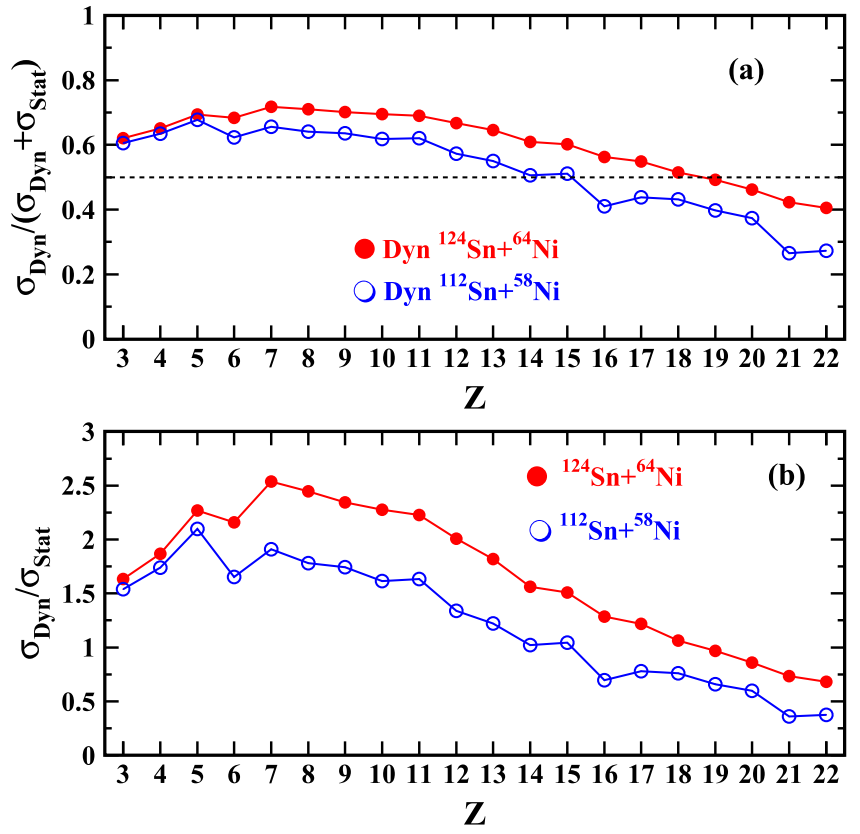

FIG. 5. (Color online) (a) Ratio of the dynamical component of the cross section to its combined (dynamical + statistical) value plotted as a function of the IMF atomic number $Z$, for the ${ }^{124} \mathrm{Sn}+$ ${ }^{64} \mathrm{Ni}$ (full circles) and ${ }^{112} \mathrm{Sn}+{ }^{58} \mathrm{Ni}$ (empty circles) systems. (b) Ratios of the dynamical-to-statistical cross sections plotted as a function of the IMF atomic number $Z$ for both $\mathrm{Sn}+\mathrm{Ni}$ systems.

from about 1.3 for light IMFs to about 2.0 for the heaviest IMFs.

It is possible to extract from Fig. 4 two additional interesting quantities: the ratio of the dynamical component of the cross section to its combined (dynamical + statistical) value and the ratio of the dynamical and statistical components. These two quantities are shown in Figs. 5(a) and 5(b) as functions of $Z$ of the IMF

It is seen that for both systems the dynamical emission prevails for light IMFs, with a maximum at $Z \approx 6-8$. A similar behavior was found in Ref. [7]. For $Z \geqslant 6$, a clear effect of enhancement of the dynamical component for the neutron-rich system is seen. With the increasing IMF's $Z$, the share of the dynamical component systematically decreases. This result is qualitatively in agreement with the stochastic mean field [29] and CoMD-II [22] calculations, both predicting an exponentially decreasing IMF-dynamical-emission probability with the IMF's size.

\section{CONCLUSIONS}

In summary, we have compared the IMF emission in semiperipheral collisions of a neutron-rich ${ }^{124} \mathrm{Sn}+{ }^{64} \mathrm{Ni}$ system and a neutron-poor system ${ }^{112} \mathrm{Sn}+{ }^{58} \mathrm{Ni}$ at the same beam energy of $35 \mathrm{~A} \mathrm{MeV}$. Generally, the IMF emission was found to be more probable for the neutron-rich system. The IMF emission was carefully studied for ternary events (with emission of only one IMF), with special emphasis on the competition between dynamical and statistical emission mechanisms. Our analysis has shown that the IMF statistical 
emission is equally probable for the two systems, while the dynamical emission is favored in case of the neutron-rich system, especially for heavier IMFs. Obviously, the effects characterizing the dynamical component of the IMF emission reflect different aspects of the underlying reaction mechanism and may depend, for example, on the parameters of the nuclear equation of state. The observed high sensitivity of the dynamical IMF emission to the $N / Z$ ratio of the colliding system confirms that isospin parameters of the equation of state could be tested using the dynamical IMF emission as an observable [12]. Specifically, the dependence of the interaction time of the colliding nuclei on the stiffness of the symmetry energy in the nuclear equation of state, evidenced by transport models $[22,30,42,43]$, may influence the competition between binary splitting and neck-fragmentation processes in heavy-ion collisions at Fermi energies. Further progress in reaction simulations for the full range of time scales (from fast dynamical emission to the equilibrated de-excitation) and for the whole IMF mass spectrum would be needed for comparisons between theory and experimental data in order to deduce the isospin dependence of the parameters of the nuclear equation of state.

A comparison of two systems having very different $N / Z$ ratios while having the same size (isobaric) would be especially attractive in that respect. Analysis of an experiment carried out at INFN-LNS in Catania on the ${ }^{124} \mathrm{Xe}+{ }^{64} \mathrm{Zn}$ system that is isobaric to the ${ }^{124} \mathrm{Sn}+{ }^{64} \mathrm{Ni}$ system is under way [44]. In that experiment a first prototype of a new particle-particle correlator (FARCOS) [45] was used to measure isotopes with high resolution in momentum space.

\section{ACKNOWLEDGMENTS}

We thank the INFN-LNS staff for providing both beams and targets of excellent quality. We are grateful also to the electronics, advanced technology, and mechanical design staffs of INFN-division of Catania. The work for V.B. was partially supported by the Romanian National Authority for Scientific Research, CNCS-UEFISCDI, Project No. PN-II-IDPCE-2011-3-0972.
[1] B. Borderie and M. Rivet, Prog. Part. Nucl. Phys. 61, 551 (2008).

[2] M. D’Agostino et al., Phys. Lett. B 473, 219 (2000).

[3] P. Chomaz, M. Colonna, and J. Randrup, Phys. Rep. 389, 263 (2004).

[4] M. D. Toro, A. Olmi, and R. Roy, Eur. Phys. J. A 30, 65 (2006).

[5] S. Piantelli, P. R. Maurenzig, A. Olmi, L. Bardelli, M. Bini, G. Casini, A. Mangiarotti, G. Pasquali, G. Poggi, and A. A. Stefanini, Phys. Rev. C 76, 061601 (2007).

[6] W. Gawlikowicz, Acta. Phys. Pol. B 40, 1695 (2009).

[7] A. B. McIntosh et al., Phys. Rev. C 81, 034603 (2010).

[8] V. Baran, M. Colonna, M. Di Toro, and R. Zus, Phys. Rev. C 85, 054611 (2012).

[9] J. Łukasik et al., Phys. Rev. C 55, 1906 (1997).

[10] P. M. Milazzo et al., Phys. Lett. B 509, 204 (2001).

[11] I. Lombardo et al., Phys. Rev. C 82, 014608 (2010).

[12] E. De Filippo et al., Phys. Rev. C 86, 014610 (2012).

[13] Z. Kohley et al., Phys. Rev. C 86, 044605 (2012).

[14] S. Barlini et al., Phys. Rev. C 87, 054607 (2013).

[15] R. S. Wang et al., Phys. Rev. C 89, 064613 (2014).

[16] M. B. Tsang et al., Phys. Rev. C 86, 015803 (2012).

[17] A. Pagano et al., Nucl. Phys. A 681, 331 (2001).

[18] A. Pagano, Nucl. Phys. News 22, 28 (2012), and references therein.

[19] A. Pagano et al., Nucl. Phys. A 734, 504 (2004).

[20] E. De Filippo et al., Phys. Rev. C 71, 044602 (2005).

[21] E. De Filippo et al., Phys. Rev. C 71, 064604 (2005).

[22] M. Papa et al., Phys. Rev. C 75, 054616 (2007).

[23] P. Russotto et al., Phys. Rev. C 81, 064605 (2010).

[24] A. A. Stefanini et al., Zeit. Phys. A 351, 167 (1995).

[25] F. Bocage et al., Nucl. Phys. A 676, 391 (2000).
[26] I. Skwira-Chalot et al., Phys. Rev. Lett. 101, 262701 (2008).

[27] J. Wilczyński et al., Phys. Rev. C 81, 024605 (2010).

[28] J. Wilczyński et al., Phys. Rev. C 81, 067604 (2010).

[29] V. Baran, M. Colonna, and M. Di Toro, Nucl. Phys. A 730, 329 (2004).

[30] V. Baran, M. Colonna, V. Greco, and M. Di Toro, Phys. Rep. 410, 335 (2005).

[31] R. J. Charity, Phys. Rev. C 82, 014610 (2010).

[32] E. De Filippo et al., Acta Phys. Pol. B 40, 1199 (2009).

[33] P. Russotto et al., in Proceedings of the International Nuclear Physics conference (INPC2007), Tokyo, June 3-8 2007, Vol. 2, edited by S. Nagamiya, T. Motobayshi, M. Oka, R. Hayano, and T. Nagae (Elsevier, Dordrecht, The Netherlands, 2008), p. 507.

[34] R. Płaneta et al., Phys. Rev. C 77, 014610 (2008).

[35] E. Geraci et al., Nucl. Phys. A 732, 173 (2004).

[36] C. Cavata, M. Demoulins, J. Gosset, M.-C. Lemaire, D. L'Hôte, J. Poitou, and O. Valette, Phys. Rev. C 42, 1760 (1990).

[37] D. Lacroix, A. Van Lauwe, and D. Durand, Phys. Rev. C 69, 054604 (2004).

[38] W. Gawlikowicz, TWI Report ZFGM-03-02 (Jagiellonian University, Krakow), 2003 (unpublished).

[39] E. De Filippo and A. Pagano, Eur. Phys. J. A 50, 32 (2014).

[40] W. W. Wilcke, Atomic Data 25, 389 (1980).

[41] V. E. Viola, K. Kwiatkowski, and M. Walker, Phys. Rev. C 31, 1550 (1985).

[42] M. Colonna, M. Di Toro, G. Fabbri, and S. Maccarone, Phys. Rev. C 57, 1410 (1998).

[43] B.-A. Li, L.-W. Chen, and C.-M. Ko, Phys. Rep. 464, 113 (2008).

[44] P. Russotto et al., J. Phys. Conf. Series 515, 012020 (2014).

[45] G. Verde et al., J. Phys. Conf. Series 420, 012158 (2013). 